\title{
R-process nucleosynthesis in a collapsar
}

\section{Shigehiro Nagataki*i}

Yukawa Institute for Theoretical Physics, Kyoto University, Oiwake-cho Kitashirakawa

Sakyo-ku, Kyoto 606-8502, Japan

E-mail: nagataki@yukawa.kyoto-u.ac.jp

\section{Shinya Wanajo}

Department of Astronomy, Graduate School of Science, The University of Tokyo, 7-3-1 Hongo,

Bunkyo-ku, Tokyo 113-0033, Japan

E-mail: wanajodastron.s.u-tokyo.ac.jp

Two-dimensional hydrodynamic simulations are performed to investigate r-process nucleosynthesis in a collapsar using the model of MacFadyen and Woosley (1999). We have solved time evolution of chemical composition with a large reaction network that contains 6000 nuclei. Nucleosynthesis calculations show that the moderately low Ye ( $=0.4$ such as in neutrino winds) results in the production of nuclei up to A 90-130. So it is concluded that more neutron-rich environment is needed to be a source of heavy r-nuclei.

International Symposium on Nuclear Astrophysics - Nuclei in the Cosmos - IX

June 25-30 2006

CERN, Geneva, Switzerland

${ }^{*}$ Speaker.

${ }^{\dagger}$ Kavli Institute for Particle Astrophysics and Cosmology Stanford Linear Accelerator Center 2575 Sand Hill Road, MS 29, Menlo Park, CA 9402 


\section{Introduction}

In the collapsar scenario, one of the most promising scenario to generate a GRB, very high entropy per baryon is considered to be realized. So unusual explosive nucleosynthesis, such as $\mathrm{R}$-process and/or $\mathrm{r} / \mathrm{p}$-process nucleosynthesis, may be realized. It is true that the event rate of a collapsar is much smaller than a normal core collapse supernova, but the products of explosive nucleosynthesis may have an influence on the chemical composition of metal poor star. This is because the jeans mass is larger at early universe, and massive stars that result in collapsars are considered to be born frequently at the early universe. In fact, recent spectroscopic analyses of extremely metal-poor stars show the large star-to-star scatter of neutron-capture element abundances (e.g., Eu; left panel). Galactic chemical evolution studies suggest that the scatter can be explained if the frequency of nucleosynthetic events are strictly limited (e.g., 8-10 $M_{\odot}$ or 25-30 $M_{\odot}$ ). The small event rate of collapsars can be a possible explanation of the large abundance scatters in the early Galaxy.

Due to the motivation mentioned above, we have performed two-dimensional hydrodynamic simulations to investigate r-process nucleosynthesis in a collapsar using the model of MacFadyen and Woosley (1999). We have also solved time evolution of chemical composition with a large reaction network that contains 6000 nuclei.

\section{Method of Calculation}

As for the progenitor model, we used the $9.15 M_{\odot}$ collapsar model of MacFadyen and Woosley (1999) with a BH of $3.762 M_{\odot}$. We have done two-dimensional hydrodynamic simulations taking account of self-gravity and gravitational potential of the central point mass. The calculated region corresponds to a quarter of the meridian plane under the assumption of axisymmetry and equatorial symmetry. The spherical mesh with $250(r) \times 30(\theta)$ grid points is used for all the computations. The radial grid is nonuniform, extending from $2.0 \times 10^{7} \mathrm{~cm}$ to $3.0 \times 10^{11} \mathrm{~cm}$ with finer grids near the center, while the polar grid is uniform. The ZEUS-2D code developed by Stone and Norman (1992a,b) has been used with an EOS of an electron-positron gas, which is in thermal equilibrium with blackbody radiation and ideal gas of nuclei [1]. Electron fraction, $Y_{e}$, is set to be 0.4 throughout of this paper since neutrino process is not included.

To realize the jet-induced explosion, we deposit only thermal energy at a rate $\dot{E}=10^{51} \mathrm{ergs}$ $\mathrm{s}^{-1}$ homogeneously within a $30^{\circ}$ cone around the rotation axis for $10 \mathrm{sec}$. In the radial direction, the deposition region extends from the inner grid boundary located at $200 \mathrm{~km}$ to a radius of $600 \mathrm{~km}$.

Since the hydrodynamics code is Eulerian, we use the test particle method [3],[4],[5] in order to obtain the informations on the time evolution of the physical quantities along the fluid motion, which are then used for the calculations of the explosive nucleosynthesis. Test particles are scattered in the progenitor and are set at rest initially. They move with the local fluid velocity at their own positions after the passage of the shock wave. The temperature and density that each test particle experiences at each time step are preserved.

Nucleosynthesis calculation for each particle is performed by solving the reaction network that consists of 6000 species from $n, p$ up to $Z=110$ isotopes. All relevant nuclear and weak reactions are included. 


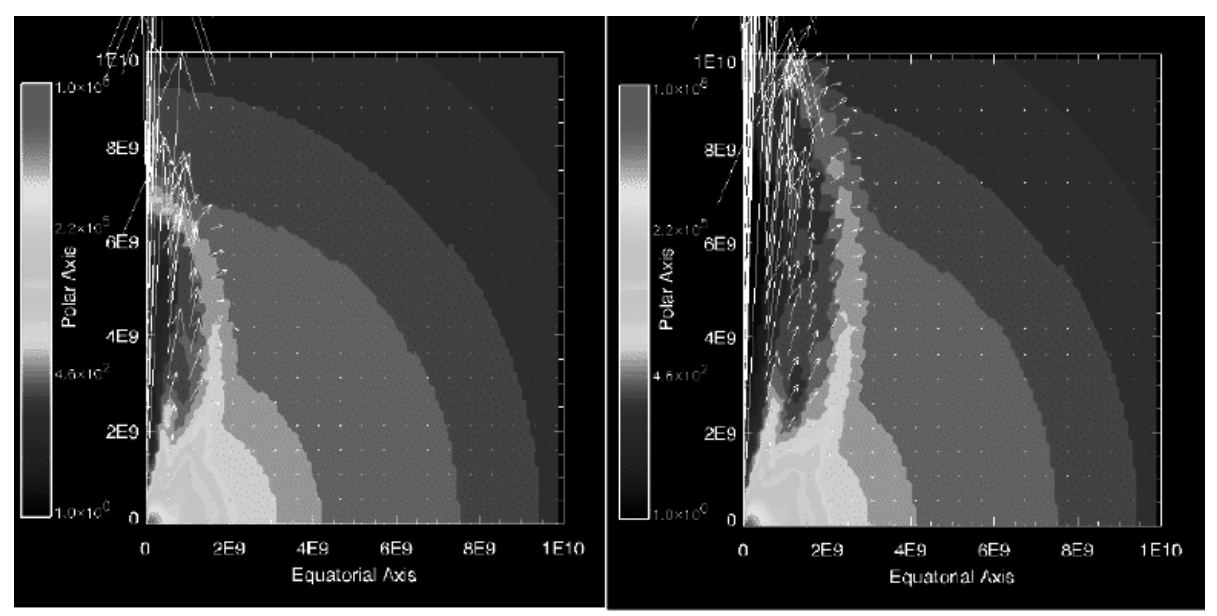

Figure 1: Density contour with velocity fields at $t=1.0 \mathrm{sec}$ (left panel) and $\mathrm{t}=1.5 \mathrm{sec}$ (right panel). Thermal energy is injected for $10 \mathrm{sec}$ to generate a GRB jet at a rate of $10^{51} \mathrm{erg} / \mathrm{s}$ at the inner most region. Due to the asymmetry of the initial density structure, the jet is formed even if only thermal energy (without kinetic energy) is deposited.

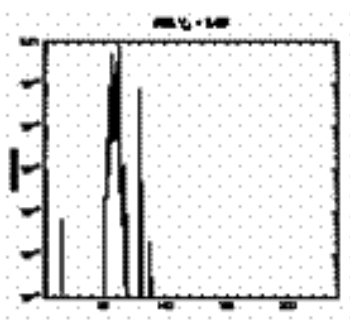

axidis

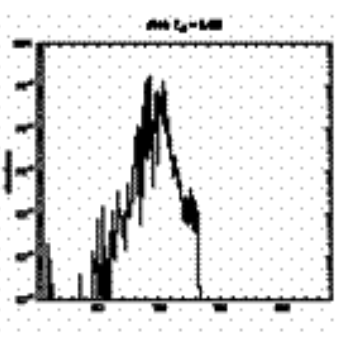

incinin
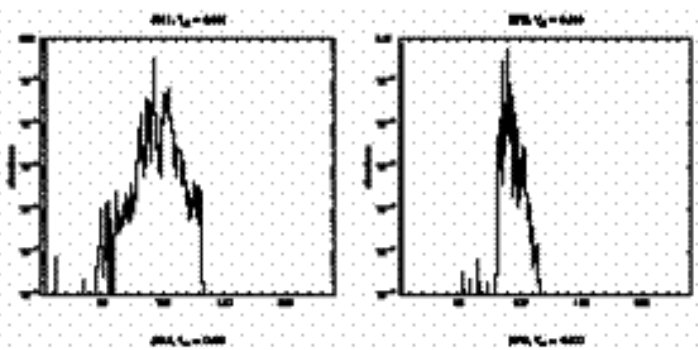

Figure 2: Nucleosynthesis results for selected test particles. The initial electron fraction is set to Ye $0=0.40$ (assuming a similar value for the late time neutrino-driven winds). Despite the high entropies for some particles, the neutron-capture proceeds only up to A 90-130.

\section{Results}

The density structure for the models in this study at $t=1.0 \mathrm{sec}$ (left panel) and $t=1.5 \mathrm{sec}$ (right panel) is shown in Fig. 1. It is clearly shown that a sharp, narrow jet propagates along to the rotation axis in Model E51, which is similar to Aloy et al. (2000). This is due to the asymmetry of the density structure of the progenitor. That is, in the low density region around the rotation axis, the injected thermal energy is mainly shared with electrons, positrons, and photons. On the other hand, at the high density region around the equatorial plane, the injected energy is shared with radiations mentioned above and nuclei/nucleons. As a result, the pressure gradient at the energy-injected region becomes aspherical, which causes a bi-polar flow along to the rotation axis.

We show results of nucleosynthesis for selected test particles in Fig. 2. Unfortunatelly, it was found that despite the high entropies for some particles, the neutron-capture proceeds only up to A 90-130.

In Fig. 3, we show the mass-averaged nucleosynthesis yields that are compared with the solar r-process abundances. It was found that the collapsars can contribute only up to A 100 nuclei for 


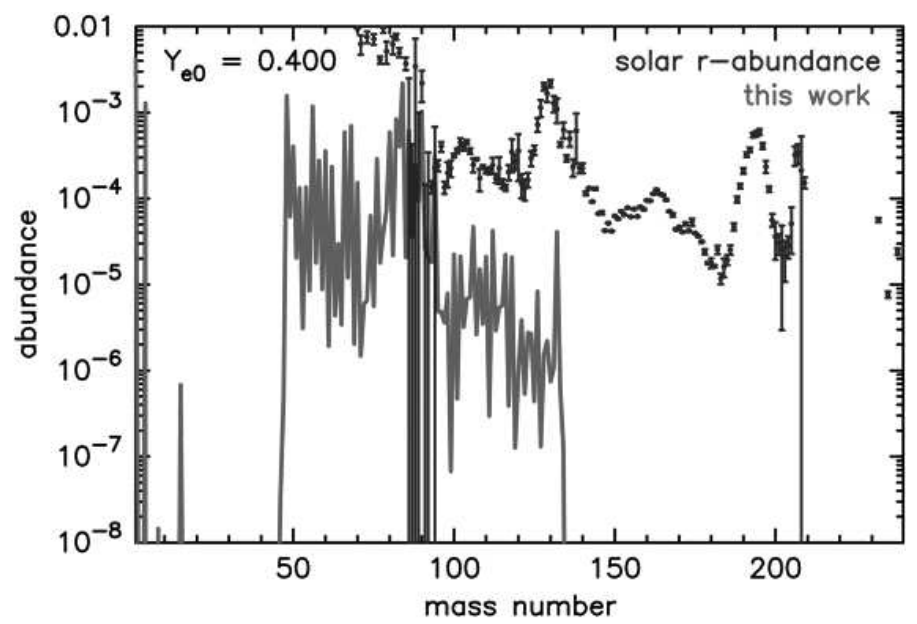

Figure 3: Mass-averaged nucleosynthesis yields are compared with the solar r-process abundances. For the choice of a moderately neutron-rich initial composition, Ye $0=0.40$, the collapsars can contribute only up to A 100 nuclei. More neutron-rich environment (e.g., $\mathrm{Ye} 0=0.30-0.20$ ) is required to be the origin of the heavy r-process nuclei.

the choice of a moderately neutron-rich initial composition, $\mathrm{Ye} 0=0.40$. So we consider that more neutron-rich environment (e.g., Ye $0=0.30-0.20$ ) is required to be the origin of the heavy r-process nuclei.

\section{Discussions}

If the jet is launched by neutrino processes, Ye may be larger than 0.5 since neutrino capture will dominate anti-neutrino capture at the jet region. This is because more neutrinos will be generated by the electron capture in the accretion disk. On the other hand, if neutrino heating process is not so efficient in the jet Region, and jet is launched by some other processes (e.g. magnetic fields), Ye will be less than 0.5. So from the observations of chemical composition in metal poor stars might reveal the environment of the central region of collapsars. This may shed light on the central engine of collapsars.

\section{Summary and Conclusion}

We have performed simulations of a collapsar, and solved time evolution of chemical composition with a large reaction network. Nucleosynthesis calculations show that the moderately low Ye (=0.4 such as in neutrino winds) results in the production of nuclei up to A 90-130. More neutronrich environment is needed to be a source of heavy r-nuclei. So we are planning to improve our numerical code so that evolution of the electron fraction of matter is followed.

\section{References}

[1] Blinnikov, S.I., Dunina-Barkovskaya, N.V., Nadyozhin, D.K. 1996, ApJ, 106, 171

[2] MacFadyen, A.I., Woosley, S.E., ApJ, 1999, 524, 262 
[3] Nagataki, S., Hashimoto, M., Sato, K., Yamada, S. 1997, ApJ, 486, 1026

[4] Nagataki, S., 2000, ApJS, 127, 141

[5] Nagataki, S., Mizuta, A., Yamada, S. Takabe, H., Sato, K. 2003, ApJ, 596, 401

[6] Stone, J.M., Norman, M.L. 1992a, ApJS, 80, 753

[7] Stone, J.M., Norman, M.L. 1992b, ApJS, 80, 791 